\title{
L'incertitude de mesure : une notion à définir en toxicologie médico-légale
}

\section{Measurement uncertainty : to be define in forensic toxicology}

\section{Christèle WIDMER-GIROD, Christian STAUB*}

Institut Universitaire de Médecine Légale, Unité de Toxicologie, 9, avenue de Champel - $\mathrm{CH}-1211$ Genève 4

*Auteur à qui adresser la correspondance : Christian STAUB, Institut Universitaire de Médecine Légale, Unité de Toxicologie, 9, avenue de Champel - CH-1211 Genève 4 - E-mail : christian.staub@medecine.unige.ch

\section{RÉSUMÉ}

L'incertitude de mesure n'est pas un concept vraiment nouveau. En effet, l'ancienne norme EN 45001 demandait déjà d'estimer ce paramètre dans les cas où cela se justifiait. Maintenant la nouvelle norme ISO 17025 va plus loin et demande que l'incertitude de mesure soit déterminée chaque fois que deux résultats sont comparés. En toxicologie médico-légale, nous nous trouvons dans cette situation:

a) lorsque nous devons rendre un résultat en nous basant sur un seuil de positivité (cut-off),

b) lors des interprétations en comparant nos résultats avec des valeurs de référence.

C'est pourquoi il est important de connaître le moyen d'estimer ce paramètre pour pouvoir l'appliquer dans le domaine de la toxicologie médico-légale où cette notion est encore peu employée

\section{MOTS-CLÉS}

Incertitude, mesure, toxicologie médico-légale.

\section{SUMMARY}

The measurement uncertainty isn't really a new concept. In fact, the former norm, EN45001, already required an estimation of this parameter in cases where it was justified. The new norm, $1 S O 17055$, goes even further and requires that the measurement uncertainty be defined each time two results are compared. In forensic toxicology, we find ourselves in this case for the following situations:

a) when we have to render a result based on a level of positivity (cut-off),

b) for interpretations when our results are compared to reference values.

That is why it is important to know the method of estimating this parameter in order to be able to apply it in the domain of forensic toxicology where this concept is still little employed.

\section{KEY-WORDS}

Uncertainty, measurement, forensic toxicology. 


\section{Introduction}

Les laboratoires d'essais doivent possếder et appliquer des procédures pour estimer l'incertitude de mesure. Une estimation raisonnable doit se baser sur une connaissance des performances de la méthode et du domaine de mesure et faire appel, par exemple, à l'expérience acquise et à des données de validation antérieures.

L'objectif d'une analyse consiste à déterminer la valeur d'une grandeur particulière à mesurer. Sachant que même dans le meilleur des cas, il subsiste toujours un doute sur la manière dont le résultat de mesure représente correctement cette valeur, le résultat d'une analyse est seulement une approximation ou estimation de la valeur de la substance à mesurer $(1,2)$.

Toutefois selon I'ILAC (The International Laboratory Accreditation Cooperation) (3), il est communément admis de donner une valeur d'incertitude dans les rapports d'analyse lorsque cette indication est utile, voire nécessaire.

\section{Détermination de 1'incerti- tude de mesure}

\section{Définition de l'incertitude de mesure (U, pour «uncertainty»)}

«L'incertitude de mesure est un paramètre associé avec le résultat d'une mesure qui caractérise la dispersion des valeurs pouvant raisonnablement être attribuée au groupe à mesurer (ou mesurande)» (4).

L'incertitude de mesure fait partie intégrante du résultat, et est une mesure de la confiance qu'on doit donner à un résultat.

Il est important de ne pas prendre le terme "incertitude" dans le sens du "doute" : au contraire, la connaissance de l'incertitude de mesure devrait impliquer une confiance plus grande dans la validité d'un résultat mesuré.

Il ne faut pas non plus confondre "erreur" et "incertitude". L'erreur est l'écart entre un résultat individuel et la valeur exacte attendue. L'incertitude de mesure prend la forme d'un intervalle et ne devrait pas être utilisée pour corriger un résultat.

D'après ISO 17025 (la nouvelle norme remplaçant l'ancienne EN 45001), tous les laboratoires d'essais doivent être capables d'évaluer l'incertitude de mesure pour chaque résultat. Le degré de rigueur de cette estimation dépend des conséquences que ce résultat pourrait avoir. En effet, si le résultat est comparé à une valeur limite ou seuil de décision (cut-off), l'incertitu- de de mesure peut avoir un rôle décisif dans l'interprétation du résultat (figure 1).

\section{Sources de l'incertitude de mesure}

Il existe de nombreuses sources de l'incertitude de mesure (3), mais essayons de citer les plus fréquentes : a) un échantillon non représentatif (la droite d'étalonnage est faite dans un milieu méthanolique alors qu'un échantillon de sang est analysé)

b) un biais lors de la lecture des instruments analogiques

c) des valeurs inexactes pour les étalons ou matériaux de référence

d) des approximations introduites dans la méthode

e) des variations entre des mesures répétées de la substance à analyser dans des conditions apparemment identiques

f) des effets de matrice

g) les conditions de conservation

h) l'échantillonnage (aliquotes)

Concentration

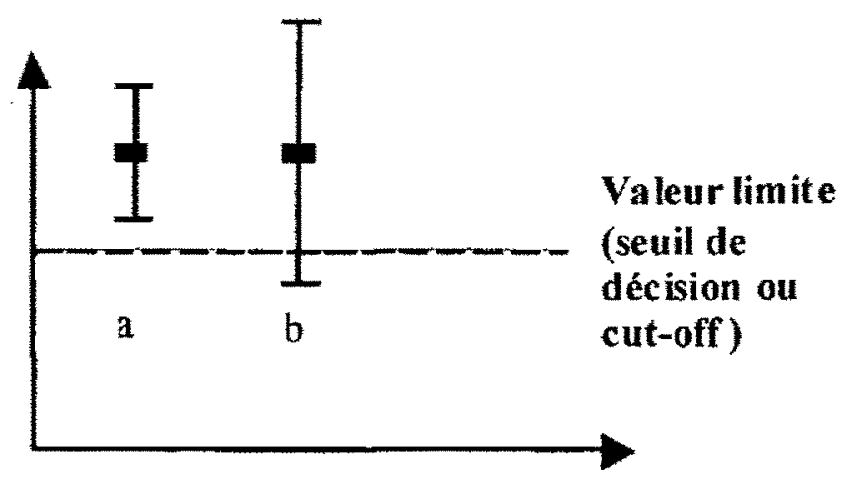

Figure I: Mesures et incertitudes en relation avec une valeur limite (d'après (5)). Dans les deux cas, la valeur moyenne est au-dessus de la valeur limite. C'est donc l'incertitude qui fait la différence. Dans le cas b, si l'incertitude de mesure est correctement déterminée, le résultat rendu pourrait être, dans certains cas, "négatif".

\section{Les différentes méthodes pour estimer l'incertitude de mesure}

Lorsqu'on estime l'incertitude de mesure, il faudrait pouvoir prendre en compte toutes les composantes de l'incertitude qui ont une importance dans l'analyse concernée.

Pour les analyses simples, il est souvent possible d'utiliser les données de validation ainsi que les données concernant les performances des méthodes pour déterminer l'U (6); nous pouvons citer :

- les données accumulées durant la validation ou la vérification de la méthode

- les données concernant les contrôles internes

- les données des essais inter- laboratoires (7). 
Pour les analyses plus complexes, il est parfois difficile d'avoir accès à tous les paramètres entrant dans la détermination de l'incertitude de mesure.

Dans ces cas, un laboratoire ayant participé à un contrôle inter-laboratoire ou contrôle de qualité externe (CQE), pourra utiliser le critère de réussite de ce contrôle comme première estimation de l'incertitude de mesure.

\section{Détermination globale de l'incertitude de mesure $(U)$}

D'après le guide ISO (1), la détermination globale de $U$ devrait tenir compte de toutes les contributions contribuant à cette dernière.

Cette détermination passe par l'estimation de l'ensemble des déviations standards (écarts-type). Toutes les composantes sont ensuite combinées à l'aide des variances. Enfin le résultat est multiplié par un facteur d'élargissement, tenant compte du niveau de confiance qu'on désire attribuer à notre mesure.

On procède généralement en plusieurs étapes (8):

a. Spécifier ce qui doit être mesuré et établir la relation mathématique entre le résultat et les données d'entrée:

$$
y=f\left(x_{1}, x_{2}, \ldots . . x_{n}\right)
$$

Il est nécessaire d'identifier toutes les sources d'incertitude en utilisant, entre autres, un diagramme de cause à effet.

Prenons l'exemple de la détermination de la densité de l'éthanol (figure 2) :

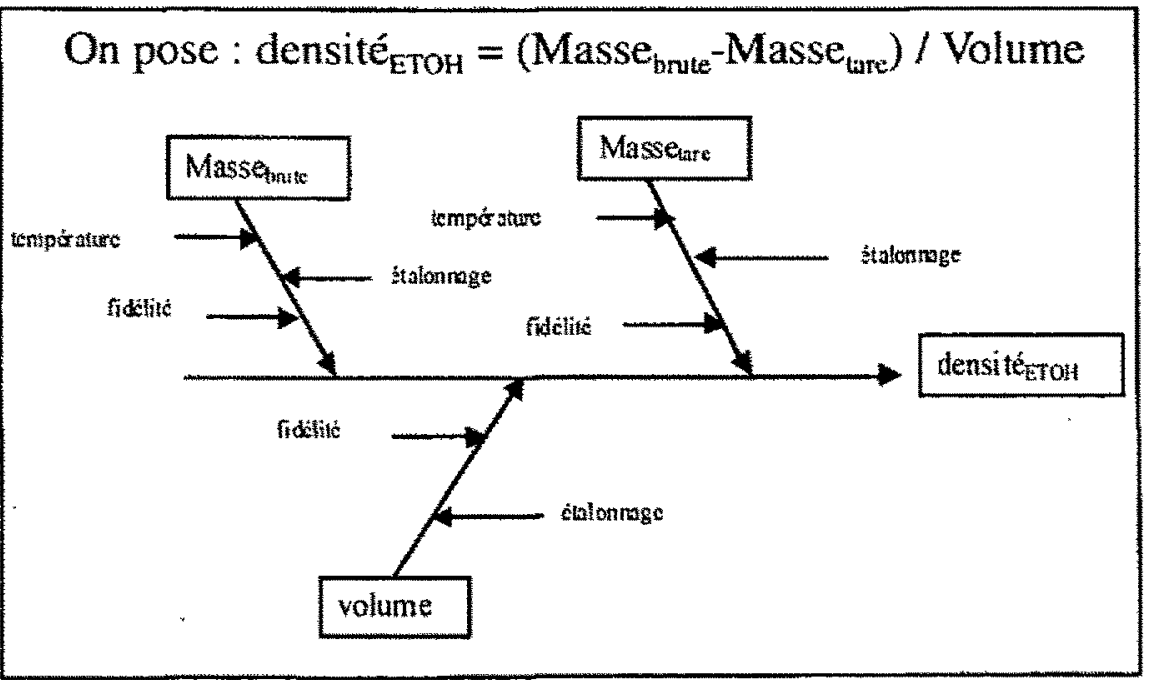

Figure 2 : Diagramme de cause à effet dans le cas de la détermination de la densité de l'éthanol.

b. Déterminer les données d'entrée $\mathrm{x}_{1}, \mathrm{x}_{2}, \ldots \mathrm{x}_{\mathrm{n}}$ (mesures, données publiées, spécifications).

c. Quantifier l'incertitude de toutes les composantes individuelles:

c1) par des moyens statistiques (données expérimentales, conditions de répétabilité) c2) de manières empirique (certificats des matériaux de référence, certificats d'étalonnage, contrôles de qualité internes et externe)

d. Calculer l'incertitude combinée uc sur la base des incertitudes des composantes individuelles déterminées selon c1 ou c2. Toutes les composantes sont exprimées sous la forme de déviation standard s (écart- type). Les variances $\left(\mathrm{s}^{2}\right)$ sont calculées et leur combinaison donne l'incertitude combinée $\boldsymbol{u}_{c}$

$$
u_{c}=\sqrt{\left(s_{1}^{2}+s_{2}^{2}\right)}
$$

où $s_{1}$ est l'écart-type d'une composante (p.e : répétabilité) et $s_{2}$ l'écart-type d'une autre composante (p.e : fidélité intermédiaire)

De manière générale, l'incertitude combinée $\boldsymbol{u}_{c}$ est un écart-type estimé qui caractérise la dispersion des valeurs qui pourrait être attribuée à la substance à analyser y. Elle sera déterminée comme étant la somme des écarts-type associés à chaque estimation.

$$
u_{c}=\sqrt{\sum_{n} s^{2}}
$$

Pour autant que les informations soient disponibles, cette méthode permet de déterminer l'ensemble des écarts-types liés aux différentes étapes de l'analyse.

e. Calculer l'incertitude élargie ou globale de mesure (U) selon le niveau de confiance choisi.

On définit $\quad U=k \times u_{c}$

où $\mathrm{k}=$ facteur d'élargissement

$(\mathrm{k}=2$ pour un niveau de confiance de $95 \%$ et 3 pour un niveau de confiance de $99 \%$ ).

Grâce à $U$, il est possible de donner, avec le résultat d'une analyse, un intervalle dont on puisse s'attendre à ce qu'il comprenne une proportion très importante des valeurs attribuables à la substance à analyser.

f. Exprimer le résultat en tenant compte de l'incertitude de mesure $(U)$

$$
\text { Résultat }=y(+/-U)
$$

dans les cas où une mesure unique a été effectuée sur l'échantillon

ou

$$
\text { Résultat }=m(+/-U)
$$

dans les cas où plusieurs mesures ont été effectuées sur l'échantillon avec

$y$ : valeur d'une mesure

$m:$ la moyenne des mesures

$U:$ l'incertitude de mesure

Cette relation permet de définir le domaine $(y(m)-U: y(m)+U)$ dans lequel la valeur de la gran- 
deur mesurée se trouve avec une probabilité de $95 \%$ (ou $99 \%$ ).

\section{Détermination de l'incertitude de mesure $(U)$ au moyen de la répétabilité et de l'écart-type.}

Lorsque toutes les informations ne sont pas disponibles, ce qui est malheureusement souvent le cas en toxicologie médico-légale, il est possible d'estimer $U$ en mesurant plusieurs fois un échantillon de contrôle ou, mieux encore, l'échantillon à analyser.

L'incertitude de mesure $(U)$ est ensuite calculée par la relation suivante :

$$
U= \pm t \times s / \sqrt{n}
$$

où :

$\mathrm{n}=$ nombre de mesures

$t=$ quantile de Student pour une probabilité de 95 ou $99 \%$ $\mathrm{s}=$ écart-type

Cette méthode ne tient pas compte des incertitudes liées aux étapes pré-analytiques (prélèvement, échantillonnage, état des échantillons) (tableau I).

Tableau I : Valeurs du quantile de Student $(t)$ en fonction du nombre de mesures.

\begin{tabular}{|c|c|c|c|}
\hline$n$ & $\begin{array}{c}\text { Niveau de confiance } \\
90 \%\end{array}$ & $\begin{array}{c}\text { Niveau de confiance } \\
95 \%\end{array}$ & $\begin{array}{c}\text { Niveau de confiance } \\
99 \%\end{array}$ \\
\hline 2 & 6,31 & 12,71 & 63,66 \\
\hline 3 & 2,92 & 4,30 & 9,93 \\
\hline 4 & 2,35 & 3,18 & 5,84 \\
\hline 6 & 2,02 & 2,57 & 4,03 \\
\hline 10 & 1,83 & 2,26 & 3,25 \\
\hline 12 & 1,80 & 2,20 & 3,11 \\
\hline 60 & 1,67 & 2,00 & 2,66 \\
\hline 9999 & 1,65 & 1,96 & 2,58 \\
\hline
\end{tabular}

\section{Estimation selon l'équation d'Horwitz (9)}

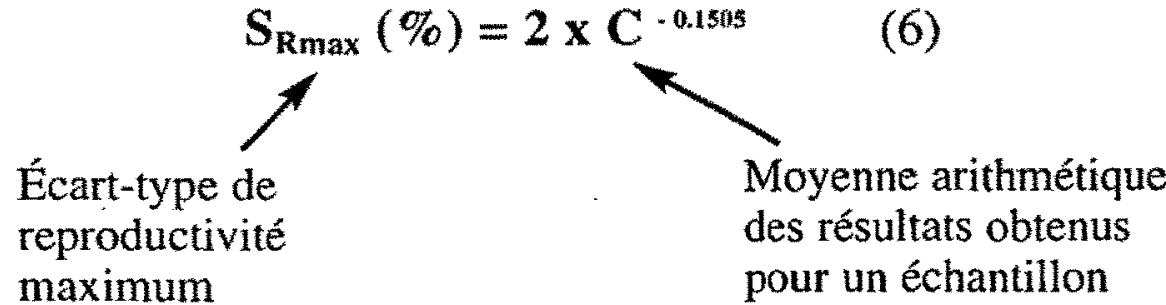

en admettant que : $\quad u_{c}=S_{R \max }$

Et en prenant la relation définie plus haut :

$$
\mathrm{U}=\mathrm{k} \times \mathrm{u}_{\mathrm{c}}
$$

avec $\mathrm{k}=2$ pour une probabilité de $95 \%$

Cette méthode ne tient compte que de la concentration et ne fait pas intervenir les paramètres propres de la méthode.
De plus, cette équation a été développée il y a quelque temps pour des applications dans le domaine alimentaire. Elle n'est plus vraiment adaptée au domaine des traces $(\mathrm{ppb})$ où une telle incertitude n'est plus acceptable $(\mathrm{U}= \pm 90 \%)$ (tableau II).

Tableau $\boldsymbol{H}$ : Estimation de l'incertitude de mesure $(U)$ selon Horwitz.

\begin{tabular}{|c|c|c|}
\hline Concentration $(\mathrm{kg} / \mathrm{kg})$ & $S_{\mathrm{R} \max }(\%)$ & Expression des résultat: \\
\hline$\%$ & 4 & $10,00 \pm 0,80 \mathrm{~g} / \mathrm{kg}$ \\
\hline$\% \circ$ & 6 & $1,00 \pm 0,11 \mathrm{~g} / \mathrm{kg}$ \\
\hline $\mathrm{mg} / \mathrm{kg}$ & 16 & $1,00 \pm 0,32 \mathrm{mg} / \mathrm{kg}$ \\
\hline$\mu \mathrm{g} / \mathrm{kg}$ & 45 & $1,00 \pm 0,90 \mu \mathrm{g} / \mathrm{kg}$ \\
\hline
\end{tabular}

\section{Application en toxicologie médico-légale}

Jusqu'à présent, très peu de laboratoires mentionnaient une incertitude de mesure dans leurs rapports d'analyse.

Cependant, alors que le nombre de laboratoires de toxicologie médico-légale accrédités est en constante augmentation, cette notion devrait, dans le futur et selon la norme 17025, figurer dans les rapports et contribuer à l'amélioration de l'interprétation des résultats.

\section{Dosage de l'éthanolémie}

En Suisse, pour avoir une valeur légale, l'éthanolémie doit être représentée par la moyenne $m$ de 4 mesures ; 2 étant obtenues par une méthode $\mathrm{A}$ et 2 par une méthode B.

Les méthodes $\mathrm{A}$ et $\mathrm{B}$ doivent être indépendantes et basées sur un principe de séparation différent (une chromatographie de partage et une chromatographie d'adsorption, par exemple).

La loi exige que le résultat de l'alcoolémie soit rendu de la manière suivante :

si $m \leq 1 \%$ :

$y=m \pm 0,05 \%$ absolu

si $\mathrm{m}>1 \%$ :

$y=m \pm 0,05 \%$ relatif

Exemples :

$0,80 \pm 0,05 \%$ ou sous la forme d'un intervalle $(0,75$ $0,85 \%$ )

$2,00 \pm 0,10 \%$ ou sous la forme d'un intervalle $(1,90$ $2,10 \%$ )

En Suisse, l'unité légale d'alcoolémie est le g/kg (ou $\%$ o) de sang complet. Ceci induit un léger bénéfice pour le conducteur dont l'éthanolémie serait mesurée en g/l.

Dans ce cas très particulier, la loi nous impose une incertitude de mesure et les laboratoires doivent contrô- 
ler que leurs mesures correspondent à l'incertitude de mesure fixée par la loi.

En utilisant la relation (5) et en tenant compte du fait que l'alcoolémie est représentée par la moyenne de quatre mesures indépendantes $(n=4)$.

On obtient : $0,05=(3,18 / 2) \times s$ d'où $s=0,0314 \%$

Le laboratoire doit donc vérifier que l'écart-type de ses mesures ne dépasse pas $0,0314 \%$.

\section{Drogues et médicaments au volant}

La Société Suisse de Médecine Légale n'impose pas d'effectuer un nombre déterminé de mesures, lorsque le laboratoire doit effectuer un dosage de drogues et/ou de médicaments dans le sang d'un conducteur de véhicule. Cependant, pour être autorisés à effectuer ce type d'analyses, les laboratoires doivent participer à des contrôles de qualité et remplir certains critères de réussite. Ces critères de réussite sont, entre autres, l'absence de faux positifs et avoir déterminé les concentrations sanguines en analytes avec une exactitude correspondant à la valeur cible plus ou moins $30 \%$.

En première approximation, on peut donc considérer que l'incertitude de mesure $(U)$ liée à ces dosages est de $\pm 30 \%$.

\section{Toxicologie post-mortem}

Il est difficile de maîtriser tous les paramètres intervenant dans l'estimation de l'incertitude de mesure. Dans les cas post-mortem, le prélèvement de l'échantillon est très important et il intervient non négligemment dans la détermination de l'incertitude. Les prélèvements se faisant en dehors du laboratoire, ce paramètre est hors contrôle. De plus, l'état de l'échantillon intervient comme source importante de l'incertitude, c'est pourquoi il est nécessaire de donner une incertitude relativement élevée.

Notre première estimation de l'incertitude de mesure $(U)$ se situe à $\pm 40 \%$ pour ce domaine.

\section{Dosage des xénobiotiques dans les che- veux}

Dans le cas des cheveux, la notion d'incertitude de mesure est encore plus récente. Son estimation est pour l'instant, comme dans les cas post-mortem, difficile.

Sur la base de contrôles inter-laboratoires, que nous avons effectués ces trois dernières années, l'incertitude de mesure $(U)$ devrait se situer autour de $\pm 50 \%$.

\section{Conclusion}

L'incertitude de mesure est un paramètre que tout laboratoire analytique devrait être en mesure de déterminer. Les laboratoires de toxicologie médico-légale ne devraient pas déroger à cette règle. En effet si, sans aucun doute, les performances de ces laboratoires se sont considérablement améliorées dans les dix dernières années ; la manière de rendre les résultats n'a pas beaucoup évolué. Il importe donc aux laboratoires, par souci de traçabilité et de transparence, de donner leurs résultats avec tout au moins une estimation de l'incertitude de mesure. En effet dans un domaine où les analyses ont des conséquences légales importantes, une meilleure connaissance du domaine de confiance entourant un résultat devrait aider l'expert dans son interprétation.

Dans ce travail, nous avons voulu présenter quelques outils statistiques, utiles à tout laboratoire analytique, que nous avons été amenés à utiliser dạns le cadre du processus d'accréditation de notre laboratoire.

\section{Références}

1. Guide to the Expression of Uncertainty in Measurement. 1993 (revised 1995) ; ISBN 92-67-10188-9.

2. General Requirements for the Competence of Testing and Calibration Laboratories. 1999 ; ISO / IEC FDIS 17025.

3. Introducing the concept of uncertainty of measurement in testing in association with application of the standard ISO / IEC 17025. ILAC-G17 : $2002 ; 1-8$.

4. International Vocabulary of Basis and General Terms in Metrology (VIM). (2nd ed.) 1993 ; ISBN 92-67-01075-1.

5. Schreiber B., Radvila P. Focal point : Analytical Technology, Quality Assurance of Analytical Data: Measurement Uncertainty and Traceability in View of ISO 17025. Chimia $2003 ; 57: 13-18$.

6. Guidelines on the Expression of Uncertainty in Quantitative Testing. 2003 ; EA-4/16 Rev.00.

7. Accuracy (trueness and precision) of Measurement Methods and Results. 1994 ; ISO/IEC 5725 : Part 1-6.

8. Rösslein M., Ellison S., Williams A. Quantifying Uncertainty in Analytical Measurement. 2000 ; Eurachem / CITAC Guide.

9. Albert R., Horwitz W. A Heuristic Derivation of the Horwitz Curve. Anal. Chem. 1997 ; 69 : 789-790. 